\title{
A Case Report: Twisted Ovarian Cyst in Pregnancy
}

\author{
Dr.Debraj Basu ${ }^{1}$, Dr.Samrat Chakrabarty ${ }^{2}$, Dr.Subrata lall Sil ${ }^{3}$,Dr.Varsha \\ $\mathrm{Saboo}^{4}$, Dr. Vidyasagar $\mathrm{Sau}^{5}$ \\ 1. DGO, MS. RMO-cum Clinical Tutor, Dept of Gynae.\& Obst.,RG Kar Medical College \\ 2. MD. Ast. Professor,Dept.of Gynae.\& Obst.. Murshidabad MC \\ 3. Professor, Dept. Of Gynae. \& Obst., R. G.Kar MC \\ 4. PG Trainee, Dept.of Gynae. \& Obst., RG Kar MC \\ 5.PG Trainee, Dept. of Pathology, RG Kar MC
}

\begin{abstract}
Ovarian torsion encountered during pregnancy carries significant risk to a pregnant woman and intrauterine foetus. In this case we report a 24 -year-old secondgravida with 14 weeks of pregnancy presenting with torsion of the left ovarian cyst. She presented to the Gynae. emergency with acute pain abdomen. She was diagnosed to have torsion of ovarian cyst with pregnancy and later emergency laparotomy followed by cystecomy was carried out. Her histopathology report showed a benign mucinous cystadenoma. Her pregnancy was followed up. She delivered a healthy female baby at term. Although the safety of antepartum surgical intervention has been accepted, abdominal surgery will always carry some risks to a pregnant woman and unborn foetus and so, before management, risks involved must be taken into consideration.
\end{abstract}

Key words: ovarian cyst torsion, pregnancy, mucinous cystadenoma

\section{Introduction}

Torsion of ovary is the total or partial rotation of the adnexa around its vascular axis or pedicle. Ovarian torsion accounts for about $3 \%$ of gynaecologic emergencies. Predisposing factors are moderate size, free mobility and long pedicle. Most common ovarian tumours with torsion are dermoid( benign cystic teratoma) and serous cystadenomas. Complete torsion causes total blockade of venous and lymphatic supply that leads to venous congestion, haemorrhage and necrosis, subsequently cyst becomes tense and may rupture. Patient usually presents with acute pain lower abdomen and pelvic examination may reveal a tender cystic mass separate from the uterus. The risk of ovarian torsion rises by 5 fold during pregnancy. Incidence is 5 per 10,000 pregnancies ${ }^{1,2}$. Torsion of ovarian tumors occurs predominantly in the reproductive age group. The majority of the cases presented in pregnant $(22.7 \%)$ than in non-pregnant $(6.1 \%)$ women $^{3}$.Here, we report a case of torsion of ovarian cyst during second trimester of pregnancy with no known predisposing factors.

\section{Case Report}

A 24 year old second gravida who delivered her first child by caesarean section 6years back presented in the emergency with pain abdomen and vomiting for one day. She was carrying a 14 weeks pregnancy and on examination her fundal height was corresponding to 14-16 weeks of gestation. Additionally a huge cystic mass felt in left lumbar region extending upto the umbilicus separate from the uterus. There was no tenderness on palpation. USG showed a single viable fetus of 14 weeks maturity and a septated cyst $(13.83 \times 7.8) \mathrm{cm}$ in left lumbar region to under the umbilicus probably originating from left ovary. She was put on a conservative management. Routine blood and urine investigations, repeat USG and serum CA125 levels(9 IU/ml) was done. Repeat USG also showed septated cystic lesion in left lumbar region which was slightly larger in size this time; $(15.0 \times 14.2 \times 9.3) \mathrm{cm}$. All other investigation reports were within normal range. As the patient was stable, laparoscopic removal of the cyst was planned but before that she again had an episode of acute pain abdomen after 7 days of admission. Diagnosing this change of symptoms as torsion of ovarian cyst emergency laparotomy had to be done. 


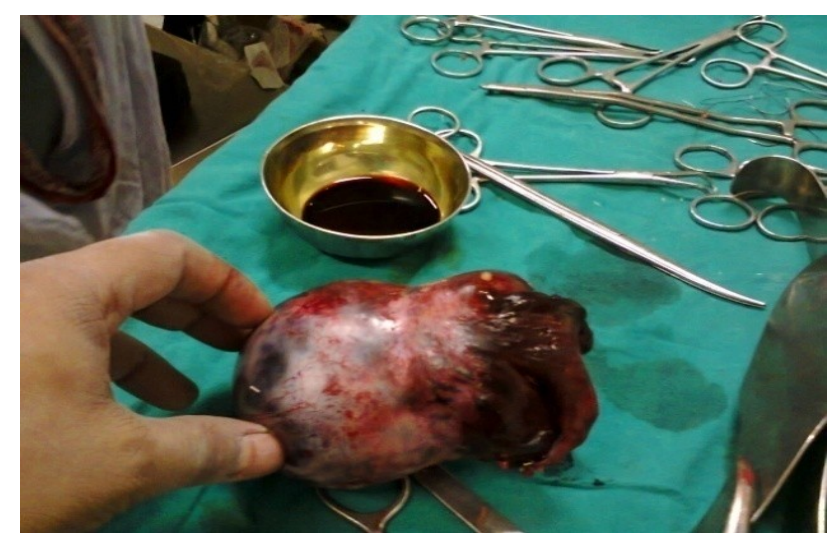

Fig.1-Ovarian cyst after torsion with gangrenous changes

During laparotomy $(15 \times 15) \mathrm{cm}$ blackened cystic mass ( Fig.1)was found in the left adnexa which was twisted around its pedicle. Left fallopian tube was found gangrenous,attached to the cyst. The cyst was cut in between the clamps, without untwisting its pedicle as it was necrosed and secured by 1-0 vicryl. Uterus was found to be 14-16 weeks in size. Right sided fallopian tube and ovary were healthy. Her post operative period was uneventful and she was discharged on $4^{\text {th }}$ postoperative day in stable condition. Histopathological examination of the specimen showed benign mucinous cystadenoma of ovary(Fig.2).

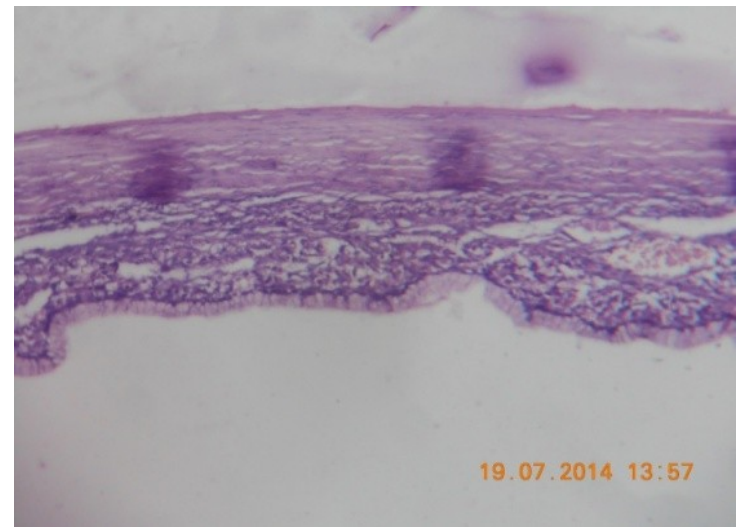

Fig.2: Histopathological section of tumour showing benign mucinous cystadenoma

She was followed up in the antenatal clinic where rest of her antenatal period was essentially uneventful. Her follow up USG scans showed normal growth and development of the fetus. She underwent an elective caesarean section at term and delivered a healthy $2.6 \mathrm{~kg}$ girl baby. Both the mother and the baby were discharged in good health on Day 7.

\section{Discussion}

The diagnosis of twisted ovarian cyst, which is an acute abdominal emergency, can be made in the majority of cases, although the symptoms are nonspecific for ovarian torsion. Changes in intra-abdominal pressure seem to start torsion in some cases, and this may account for cases seen during pregnancy or the puerperium. The usual symptoms of torsion of an ovarian cyst are acute, violent, colicky pains in the lower abdomen, distention and nausea, often with vomitting. Abdominal tenderness is usually present, and localized over the site of the cyst, although sometimes it become generalized. Abdominal rigidity is also found in most cases indicating either the blood supply of the ovary is involved or that haemorrhage has taken place. To make a correct diagnosis of twisted ovarian tumour speacially in pregnancy ultrasonographic confirmation is needed. When pregnancy is present ovarian cyst usually is found alongside pregnant uterus,though presence of abdominal rigidity make palpation of such a mass difficult. The use of colour Doppler sonography, with the main sign of the absence of intraparenchymal ovarian blood flow indicative of adnexal torsion, a decreased blood flow if present can be a result of incomplete torsion.

The commonest type of ovarian tumours in pregnancy are cystic teratoma, paraovarian cysts,serous cystadenoma,corpus luteal cysts etc ${ }^{1,3}$. In this case however the histopathology reveals mucinous cyst adenoma which is very uncommon for ovarian torsion even in non-pregnant condition. About 15-20\% of all ovarian 
tumors are mucinous cystadenomas. They often become very large and can fill up the entire abdomen and are usually multi-septated, cystic masses with thin walls and contain varying amounts of solid tissue. Benign mucinous cystadenomas compose $80 \%$ of mucinous ovarian tumors ${ }^{4}$ and $20-25 \%$ of benign ovarian tumors overall. The peak incidence occurs between 30-50 years of age. Benign tumors are bilateral in 5-10\% of cases.

Differential diagnosis of ovarian cyst torsion in pregnancy includes: uterine leiomyomas, non preganant horn of bicornuate uterus, appendiceal abscess, diverticular abscess, pelvic kidney, retroperitoneal tumours, ectopic pregnancy and retroverted gravid uterus ${ }^{3}$. Complications of the ovarian cysts in pregnancy are torsion of the cyst, rupture, infection, malignancy, impaction of cyst in pelvis causing retention of urine, malpresentation of foetus and during labour obstructed labour ${ }^{3}$. Most ovarian masses can either remain uneventful or resolve throughout pregnancy and that the incidence of the above risks are actually $\operatorname{low}^{5,6}$. Ovarian torsion, occurs most frequently in the first trimester, occasionally in the second, and rarely in the third ${ }^{7}$.

Cysts $\mathrm{with}$ less than $6 \mathrm{~cm}$ diameter and appearing benign on ultrasound are generally treated conservatively as mostly they undergo spontaneous resolution. Corpus luteal cysts regress by 12 to 16 weeks ${ }^{1,8}$. Cysts more than 10 centimetres in size are usually resected due to increased risk of torsion, rupture or malignancy and laparoscopic removal is recommended. However they may require emergency exploratory laparotomy for rupture, torsion or infarction in as many as $50 \%$ cases ${ }^{4}$.

If the ovarian cyst is diagnosed in the first trimester, it is better to wait till 16 wks when the implantation of pregnancy is more secure and also the cyst may disappear spontaneously. Persisting tumours are treated by cystectomy or ovariotomy as indicated. Ovarian tumour or cyst can be easily removed till 28 wks of gestation thereafter not only it becomes hard to access but also operation may precipitate preterm labour. Ovarian cyst if ruptures, or undergoes torsion or if it shows evidence of malignancy, immediate surgery is needed, irrespective of the period of gestation ${ }^{4}$.Previously untwisting of the pedicle was avoided to prevent emboli and toxic substances related to hypoxia, from entering peripheral circulation, but recently, re-establishing ovarian circulation by untwisting, has shown to improve circulation in viable ovarian tissue with no systemic complications ${ }^{1,2}$. Obviously in cases where ovarian cysts have undergone gangrenous changes, untwisting are not tried, which was present in the present case.

\section{Conclusion}

In this case an early diagnosis might have helped to conserve patient's adnexa. Though it is an extremely rare problem in pregnancy, adnexal torsion should be taken into consideration in the differential diagnosis of abdominal pain. Treatment options are limited to surgery, either by laparoscopy or laparotomy, but the former becomes more difficult after second trimester. In our case we performed a laparotomy with Pfannenstiel incision, and did not attempt to untwist the adnexa because of widespread necrosis?.

\section{References}

[1]. Kolluru V, Gurumurthy R, Vellanki V, Gururaj D.Torsion of ovarian cyst during pregnancy: a case report.Cases Journal 2009;2:9405. http://dx.doi.org/10.1186/1757-1626-2-9405 PMid:20090873 PMCid:2809077

[2]. Ventolini G, Hunter L, Drollinger D, Hurd WW: Ovarian torsion during pregnancy. [http://www.residentandstaff.com/issues/arti cles/2005-09 04.asp].

[3]. Lee $\mathrm{CH}$, Raman S, Sivanesaratnam V:Torsion of ovarian tumors:a clinicopathological study.Int JGynaecol Obstet1989, 28:21-25.

[4]. Hart WR (January 2005). "Mucinous tumors of the ovary: a review". Int. J. Gynecol. Pathol. 24 (1): 4- 25. PMID 15626914

[5]. Yen CF, Lin SL, Murk W, Wang CJ, Lee CL, Soong YK, Arici A: Risk analysis of torsion and malignancy for adnexal mases duringpregnancy. Fertil Steril 2009, 91(5):1895-902.

[6]. Schmeler KM, Mayo-smith WW, Peipert JF, Weitzen S, Manuel MD,Gordinier ME: Adnexal masses in pregnancy: surgery compared with observation. Obstet Gynecol 2005, 105:1098-103.

[7]. Hibbard LT: Adnexal torsion. Am J Obstet Gynecol 1985,152:456-461

[8]. Duic Z, Kukura V, Ciglar S, et al.: Adnexal masses in pregnancy:a review of eight cases undergoing surgical management. Eur J Gynaecol Oncol 2002, 23:133-134.

[9]. E. KARAALp , N.YUcEL, F. DEMIRcI , E. AYDIN, B. KARAKOc : Adnexal torsion in a first-trimester pregnant patient without any predisposing factor: A case report; Göztepe Tip Dergisi 28(1):58-60, 2013 doi:10.5222/J.GOZTEPETRH.2013.058 